\title{
3D continuum radiative transfer
}

\section{A new adaptive grid construction algorithm based on the Monte Carlo method}

\author{
G. Niccolini and J. Alcolea
}

\author{
Observatorio Astronómico Nacional (IGN), Alfonso XII 3, 28014 Madrid, Spain \\ e-mail: g.niccolini@oan.es
}

Received 25 October 2005 / Accepted 12 May 2006

ABSTRACT

\begin{abstract}
Aims. The continuum radiative transfer is a common problem in Astrophysics, that must be solved to describe the physics and interpret the observations of objects embedded (for instance) in a dusty medium (e.g. evolved stars, young stellar objects, ...). Extreme high angular resolution observations reveal in general complex geometries (e.g. mediums with clumps of matter, warps ...). It is thus necessary to solve the radiative transfer problem making no simplifying assumptions on the geometry, i.e. resolve the full spatial three dimensional problem.

Methods. We use the flexible Monte Carlo (MC) method to solve the continuum radiative transfer problem. We focus on the computation of the temperature structure of a dusty medium and the construction of the grid that samples this structure. We propose to use a natural by-product of the MC method, the locations of the photon package absorptions in the medium, to build an adaptive grid to reach the finest resolution in the regions of interest.

Results. We present the details of the new algorithm used for the construction of the grid. We also propose modifications to a widely used MC propagation framework. The reliability of our method is numerically tested against well established numerical results in one and two dimensions. In order to illustrate the capabilities of our three dimensional methods, we examine the observational evidence for the presence of warps in circumstellar discs.
\end{abstract}

Key words. radiative transfer - methods: numerical - stars: AGB and post-AGB - stars: planetary systems: protoplanetary disks

\section{Introduction}

The resolution of the continuum radiative transfer is a common problem in many fields in astrophysics. For instance, there is a wide variety of objects showing continuum dust grain emission: circumstellar discs around Young Stellar Objects (YSOs), evolved stars, active galactic nuclei ... With the high angular resolution of present ground-based and space instruments such as the Very Large Telescope Interferometer or the Hubble Space Telescope (HST), or of next decade instruments such as the Atacama Large Millimeter Array (ALMA) or the Next Generation Space Telescope (NGST), astrophysical objects reveal or are expected to reveal complex structures and geometries: clumps around R Coronae Borealis variable ( $\mathrm{RCrB})$ stars (de Laverny \& Mékarnia 2004), gaps in YSO circumstellar discs (Wolf \& D'Angelo 2005), warped discs (see Heap et al. 2000 , for the $\beta$-Pictoris case) $\ldots$ The need to solve the 3D radiative transfer is twofold: firstly, we must interpret these high angular observations in order to characterise the observed object; second, we must be able to describe the physics of these objects for which radiative transfer is a crucial ingredient.

The Monte Carlo (MC) method is a flexible way to solve the radiative transfer problem. It is flexible in the sense that no particular assumptions must be made on the medium opacities or on the geometry of the considered problem. The main drawback of the method, i.e. the slowness of execution, can be compensated by parallel computing and proper weighting (e.g. Watson \& Henney 2001; Juvela 2005) of the test particles.

In this paper we focus on radiative transfer through dusty media. On the one hand, the aim is to describe the radiation field, with the help of the Monte Carlo simulation. This is relatively easy for a pure scattering medium, and the first attempts date back to the 70 for the first astrophysical applications (Mattila 1970; Roark et al. 1974; Witt 1977). On the other hand, radiation can be absorbed and re-emitted by dust grains whose temperatures are determined (neglecting dust-gas coupling) by the equilibrium between absorbed and emitted energy. This situation is far more complicated since we must face the coupled problems of the simultaneous determination of the radiation field and the temperature structure of the dusty medium. Indeed, to solve this problem common techniques involve iterations on the dust grain emission and/or determination of the temperature via iterative techniques (or variations on this theme), until the condition of radiative equilibrium is reached to a certain accuracy. This problem has been tackled for some simple geometries (Lefevre et al. 1982, 1983) and reached more complex geometries (2-3D) following the increasing computing capabilities (e.g. Lopez et al. 1995; Lucy 1999; Wolf et al. 1999; Misselt et al. 2001; Niccolini et al. 2003; Juvela \& Padoan 2003).

The method proposed by Bjorkman \& Wood (2001, BW in the following) departs from these common techniques. Within the framework of this method, photon packages of fixed energy are emitted immediately after each absorption, ensuring radiative equilibrium during the Monte Carlo sampling of the radiation field. This feature is common to the method used by Lucy (1999) but BW introduced a correction in the frequency distribution of the emitted photons, since photon packages are emitted according to temperatures departing from the final solution. The validity of this Frequency Distribution Adjustment (FDA) was demonstrated by Baes et al. (2005). As a consequence of this method, the radiative equilibrium solution for the temperatures is obtained after the propagation of all photon packages, without 
the need for further iterative procedures. Still, the determination of the local temperature after each absorption does require, of course, an iterative procedure.

We propose to modify the BW method for the computation of the equilibrium temperature structure. Indeed, it is possible to obtain an estimation of the final solution for the temperature structure, even before all photon packages considered in the Monte Carlo simulation have been propagated (with the same level of Monte Carlo random noise). With the BW method, the temperature structure relaxes to the radiative equilibrium only at the very end of the simulation, otherwise being lower than the final solution (since the medium is heated by radiation). We propose a modification that makes it possible to reach an acceptable solution much faster during the simulation. This can be useful while fitting some observations in very time consuming cases, where the parameters of a particular model must be varied by hand. We also generalised this method to many photon sources. We show that the self-absorption within the volume element sampling the problem must be taken into account with special care when dealing with more than one radiation source.

We also propose to make use of a natural by-product of the Monte Carlo simulation: the locations of the absorption of the photon packages, to generate a grid sampling the temperature structure. With this knowledge, it is possible to generate an adaptive grid by concentrating the cells in the regions where the number of absorptions/emissions is larger. In this way, the use of the computer memory is optimised in the sense that the cells are concentrated in the regions of interest from the point of view of the temperature determination, a feature common to all adaptive grids. The main interesting feature of this new technique is that no further computation is needed to build the grid since its construction can be undertaken during the propagation of the photon packages, avoiding the computation of the grid before starting the resolution of the radiative transfer problem.

In Sect. 2 we describe the Monte Carlo simulation that we have used and the modified version of the temperature determination of BW we propose. In Sect. 3 the algorithm for the grid construction is presented. Numerical tests of our radiative transfer code against well established benchmarks in one and two dimensions (Ivezic et al. 1997; Pascucci et al. 2003) are shown in Sect. 4. We also present numerical tests of the temperature determination method and the grid construction algorithm from the point of view of the precision (the Monte Carlo noise) and the accuracy (the level of discrepancy with reference solutions).

In Sect. 5, we use the ability of our code to deal with full 3D problems to solve the continuum radiative transfer problem in the case of a warped accretion disc. There is observational (e.g. Heap et al. 2000; Matsuura et al. 2005) and theoretical (e.g. Pringle 1996, 1997) evidence that accretion discs generally reveal warps in many astrophysical objects: active galactic nuclei, neutron stars, planetary nebulae, Xray binaries, etc.

\section{Radiative transfer in 3D}

We present the MC method used for the propagation of the photon packages, complemented with the temperature determination algorithm (under the LTE assumption) based on a modification of the BW method. Special treatments necessary to take many photon package sources into account are also described.

\subsection{Temperature sampling grid}

The model volume is subdivided into several volume elements, called cells. The whole set of cells is called the temperature sampling grid or the grid. Note that we do not assume that the density of dust grains (or any other quantity but the temperature) is constant within a cell. For the moment, we make no assumptions on the particular grid that is used; we simply identify each cell of the grid by an index $\xi$. Note that with the grid construction algorithm presented in Sect. 3.1, the number of cells is not necessarily fixed during the propagation of the photon packages (see below).

\subsection{Opacities}

We consider a model volume filled with a medium characterised by its opacities, $\kappa_{v}^{\mathrm{ext}}=\kappa_{v}^{\mathrm{abs}}+\kappa_{v}^{\mathrm{sca}}, \kappa_{v}^{\mathrm{abs}}(\boldsymbol{r})$ and $\kappa_{v}^{\mathrm{sca}}(\boldsymbol{r})$, being the extinction, absorption and scattering coefficients at frequency $v$, expressed in $\mathrm{m}^{-1}$. The scattering, supposed to be coherent, is additionally described by the phase function $p_{v}\left(\hat{n}, \hat{n}^{\prime}, \boldsymbol{r}\right)$, giving the fraction of radiant energy scattered at point $\boldsymbol{r}$ from the direction $\hat{n}$ in the direction $\hat{n}^{\prime}$.

For simplicity, we will make some supplementary assumptions:

- the opacities are temperature independent. For instance, this can represent dust opacities within the limit of the Mie theory;

- the cross sections and the phase function do not depend on the spatial location, i.e. they are uniform through the model volume. However, the methods we present here are not restricted to this special case, used only to simplify our considerations. Also note that $\kappa_{v}^{\text {abs }}, \kappa_{v}^{\text {sca }}, \kappa_{v}^{\text {ext }}$ do have spatial variations through the model volume via the variation of the number density of scatterers/emitters. Notice that the number density is in general not constant through a cell of the temperature grid. The number density can be provided through an analytical expression (e.g. via a computer program function) or be sampled on a spatial grid of its own.

\subsection{Propagation algorithm}

The propagation algorithm we use to describe the radiation is summarised by the following sequence (a detailed description can be found for instance in Wolf et al. 1999):

1. Each source emits its own set of $N_{k}$ photon packages. The choice of the emission direction and frequency is done in a random manner, weighted by the emitting properties of the source (e.g. see Cashwell \& Everett 1959).

2. A photon package travels through the medium over an optical depth $\tau_{v}$, where $v$ is the frequency of the photon packages. The random choice of $\tau_{v}$ is chosen according to the Probability Density Function (PDF) $\mathrm{e}^{-\tau_{\nu}}$.

3. The photon package, now "located" at the position of the interaction, has an interaction with the medium. The albedo $\gamma_{v}=\frac{\kappa_{v}^{\text {sa }}}{\kappa_{v}^{\text {ext }}}$, gives the probability that the photon package is scattered rather that absorbed.

- if the photon package is scattered, we choose a new direction of propagation according to the phase function $p_{v}$;

- if the photon package is absorbed, we emit a new photon package at a new frequency $v^{\prime}$ from the same position. $v^{\prime}$ is selected according to the new estimate of the local temperature derived from Eq. (5). The new frequency is chosen according to Eq. (6).

4. This procedure is repeated from point 2 until the photon package exits the model volume and contributes to the 
outgoing flux. Then the procedure is carried on from point 1 for all sources in the problem.

By storing the outgoing photon package directions and energies, it is possible to derive the emitted fluxes and build images at any frequency or the Spectral Energy Distribution (SED).

\subsection{Radiative equilibrium temperatures}

The method of BW allows one to compute the radiative equilibrium temperatures while the photon packages are propagating through the medium. The method presented in this section differs from BW in three points :

1. we take into account several sources of photon packages;

2. we propose another estimator for the temperatures, and as a consequence no FDA is necessary, as in the BW method;

3 . the self-absorption within the cells requires special care when dealing with two or more sources having photon packages of different energies.

\subsubsection{Generalisation to $K$ sources}

We assume that we have $K$ sources of photon packages, characterised by their luminosities $L_{k}$, for $k=1, \ldots, K$. The energy $E_{k}$ of a photon package emitted by the source $k$ is defined by

$E_{k}=\frac{L_{k} \Delta t}{N_{k}}$

where $\Delta t$ is the time interval considered and $N_{k}$ is the number of photon packages emitted by this particular source.

The energy emitted by the cell $\xi, E^{\mathrm{em}}$ is given by

$$
\begin{aligned}
E_{\xi}^{\mathrm{em}} & =4 \pi \Delta t \iiint_{\mathcal{V}_{\xi}} \int_{0}^{\infty} n(\boldsymbol{r}) C_{v}^{\mathrm{abs}} B_{v}\left(T_{\xi}\right) \mathrm{d} v \mathrm{~d}^{3} \boldsymbol{r} \\
& =4 \pi \Delta t n_{\xi} C_{\mathrm{P}}^{\mathrm{abs}}\left(T_{\xi}\right) B\left(T_{\xi}\right),
\end{aligned}
$$

where $\mathcal{V}_{\xi}$ and $T_{\xi}$ are respectively the volume and the temperature of cell $\xi, n(\boldsymbol{r})$ is the number density of scatterers/emitters at position $\boldsymbol{r}$ and $n_{\xi}$ its volume integral over $\mathcal{V}_{\xi} \cdot C_{v}^{\text {abs }}$ and $C_{\mathrm{P}}^{\text {abs }}$ are respectively the absorption cross-section and the Planck mean of the absorption cross-section, $B_{v}$ the Planck function and $B\left(T_{\xi}\right)$ its integral over the frequencies, i.e. $\frac{\sigma}{\pi} T_{\xi}^{4}$.

The energy $E_{k, \xi}^{\text {abs }}$ emitted by the source $k$ and absorbed by the cell $\xi$ is given by

$E_{k, \xi}^{\mathrm{abs}}=N_{k, \xi}^{\mathrm{abs}} E_{k}$,

where $N_{k, \xi}^{\text {abs }}$ is the number of photon packages emitted by the source $k$ and absorbed by the cell $\xi$. Note that this quantity is a random variable computed during the $\mathrm{MC}$ simulation.

Under the assumption of radiative equilibrium, locally, the energy absorbed by the dusty medium compensates exactly the energy emitted. From Eqs. (1), (2) and (3) we obtain

$\sigma T_{\xi}^{4}=\frac{\sum_{k=0}^{K} \frac{N_{k, \xi}^{\mathrm{abs}}}{N_{k}} L_{k}}{4 n_{\xi} C_{\mathrm{P}}^{\mathrm{abs}}\left(T_{\xi}\right)}$.

From Eq. (4), we can derive the temperature of the cell $\xi$ each time a new photon package is absorbed. Note that $T_{\xi}$ is not the solution (to a certain level of accuracy) of the problem until all the photon packages heat up the medium. As a consequence, a special correction procedure of the flux must be done (see Eq. (7) in Bjorkman \& Wood 2001). In the next section, we proceed to a modification of Eq. (4).

\subsubsection{An improved temperature estimation}

With Eq. (4), i.e. using the method of BW, the temperature structure will relax to the radiative equilibrium solution to a certain accuracy, only after the whole set of $N_{k}$ photon packages for the $K$ sources considered in the problem are propagated through the medium. In the process, the temperature structure and the flux could differ substantially from the final estimation.

One consequence of the BW method is that the estimation of the temperature is only obtained at the very end of the computation. However, while fitting some observational data, it would be helpful to be able to have an hint as to what the final results will be, with the accuracy given by the number of photon packages that have already been used up to that point. Hereafter, we propose modifications to the BW scheme to obtain such a prediction.

An other estimator of the temperature can be obtained by correcting the fact that only $n_{k}$ photon packages have been propagated (for source $k$ ) at some particular point during the MC simulation. It is possible to estimate what $N_{k, \xi}^{\text {abs }}$ would be for a particular cell $\xi$, after the $N_{k}$ photon packages have heated up the medium. This is simply given by $N_{k, \xi}^{\text {abs }} \times \frac{N_{k}}{n_{k}}$. Applying this correction, the new estimator of the temperature when only $n_{k}$ photon packages have been propagated is

$\sigma T_{\xi}^{4}=\frac{\sum_{k=0}^{K} \frac{N_{k, \xi}^{\mathrm{abs}}}{n_{k}} L_{k}}{4 n_{\xi} C_{\mathrm{P}}^{\mathrm{abs}}\left(T_{\xi}\right)}$.

Again, the temperature or the flux determined from Eq. (5) very rapidly give an accurate estimation of the solution while the determination through Eq. (4) requires almost the whole set of photon packages to be emitted (see Sect. 4.3).

Once the temperature is computed from Eq. (8), the probability $p_{v} \mathrm{~d} v$ of emitting a photon with a frequency between $v$ and $v+\mathrm{d} v$, within cell $\xi$, is simply

$p_{v} d v=\frac{\kappa_{v}^{\mathrm{abs}} B_{v}\left(T_{\xi}\right) \mathrm{d} v}{\int_{0}^{\infty} \kappa_{v}^{\mathrm{abs}} B_{v}\left(T_{\xi}\right) \mathrm{d} v}$

Note that for BW this probability is proportional to $\approx$ $\kappa_{v}^{\mathrm{abs}} \partial_{T} B_{v}\left(T_{\xi}\right)$

However, it is not obvious that using Eqs. (5) and (6) one obtains estimators for the temperature and the flux. Indeed, it can be shown that the flux and the temperature of an isolated cell estimated in that way are convergent (in probability) estimators. It is beyond the scope of this paper to present such a proof and this will be the subject of a subsequent paper. However, we present in Sect. 4.3 a study of the numerical quality of the estimators compared to the BW alternatives.

\subsubsection{Treatment of self-absorption}

For any cell there is a finite probability that a photon package emitted within its volume is re-absorbed in the same volume. Of course, this self-absorption within the cell must not be counted as an energy gain for it. As a first consequence, in Eq. (3), $N_{k, \xi}^{\text {abs }}$ must not include self-absorption. As a second consequence, Eq. (2) must be corrected as well. Indeed, $E^{\mathrm{em}}$, as expressed in Eq. (2), is the energy emitted and thus exiting cell $\xi$. However, only a given fraction of the photon packages emitted within $\xi$ manage to exit the cell; the remaining fraction is absorbed within $\xi$ itself and must be subtracted from $E^{\mathrm{em}}$. We define $N_{k, \xi}^{\mathrm{sabs}}$ as the number of self-absorptions within the cell $\xi$ for photon packages 
emitted by the source $k$. The volume integral of the number density, $n_{\xi}$, must be corrected to take into account that only the estimated fraction $\frac{N^{\text {abs }}}{N^{\text {abs }}+N^{\text {sabs }}}$ of $\mathcal{V}_{\xi}$ contributes to the exiting energy from the cell. This leads to the corrected expression for $E^{\mathrm{em}}$

$E_{\xi}^{\mathrm{em}}=4 \pi \Delta t n_{\xi} \frac{\sum_{k=0}^{K} N_{k, \xi}^{\mathrm{abs}}}{\sum_{k=0}^{K}\left(N_{k, \xi}^{\mathrm{abs}}+N_{k, \xi}^{\mathrm{sabs}}\right)} C_{\mathrm{P}}^{\mathrm{abs}}\left(T_{\xi}\right) B\left(T_{\xi}\right)$,

and the temperature is consequently determined from

$$
\sigma T_{\xi}^{4}=\frac{\sum_{k=0}^{K} \frac{N_{k, \xi}^{\mathrm{abs}}}{n_{k}} L_{k}}{4 n_{\xi} \frac{\sum_{k=0}^{K} N_{k, \xi}^{\mathrm{abs}}}{\sum_{k=0}^{K}\left(N_{k, \xi}^{\mathrm{abs}}+N_{k, \xi}^{\mathrm{sabs}}\right)} C_{\mathrm{P}}^{\mathrm{abs}}\left(T_{\xi}\right)},
$$

from which we obtain the estimator $T_{\xi}$ of the temperature for many sources, taking correctly into account self-absorption.

When the ratio $\frac{L_{k}}{N_{k}}$ is independent of $k$, i.e. independent of the source, Eq. (8) reduces to Eq. (5), where self-absorption is not taken into account, i.e. $N^{\text {abs }}$ is actually considered to be $N^{\text {abs }}+$ $N^{\text {sabs }}$. In that case, the expressions for $E^{\mathrm{em}}$ and $E^{\mathrm{abs}}$ would be wrong but the two errors cancel each other to give the correct equation for the temperature.

Note that considering only one single source of photon packages, one falls in this special case. As long as one is not interested in the exact values of $E^{\mathrm{em}}$ and $E^{\mathrm{abs}}$ the special treatment of self-absorption could be forgotten if $\frac{L_{k}}{N_{k}}$ is constant. However, one might obtain bad statistical representation of the sources since the larger the luminosity, the larger must be the number of photon packages to ensure a constant $\frac{L_{k}}{N_{k}}$.

\section{Adaptive grid construction}

In this section we propose an algorithm for the grid construction based on the number of absorptions of photon packages packages by the medium: whenever the number of absorptions in a particular cell exceeds a prescribed value, the cell is divided into sub-cells. This kind of procedure was adopted by Harries et al. (2004), using a similar refinement procedure but based on the density of a ballistic particle model, for the particular object they studied, rather than on the photon package absorptions. The cells are identified via a tree-code search algorithm.

\subsection{Construction algorithm}

The interactions (in particular the absorptions) of the photon packages with the medium control completely the MC simulation, even when the number of absorptions is not used to determine the temperature (see Lucy 1999) and might appear as a secondary by-product. To build the grid, we will make use of this natural information provided by the MC method.

We present the construction algorithm for a 3D Cartesian grid (the cells are cubes) but the following considerations are not restricted to any particular geometry and can be easily extended to other cases. The construction algorithm can be depicted as follows:

- We first initialise the algorithm by enclosing the considered model volume in a first cell, taken sufficiently large.

- While proceeding to the propagation of photon packages, we count the number of absorptions within each cell and store their locations. This storage is only needed during a fraction of the total computing time until the number of cell in the grid reaches a prescribed value $N_{\text {cells }}$. At this point, the memory can be released. For all optically thick examples presented in Sects. 4 and 5, less than a few $10^{2} \mathrm{MB}$ were necessary (for floating numbers coded with 8 bytes).

- If it happens that the number of absorptions in one cell after an absorption event is greater than a specified number $N_{\max }$, we split the cell into sub-cells in a way that depends on the particular geometry of the problem. For the special case we consider here, the cell is split in the 8 sub-cubes it contains. We compute the temperature of these freshly created sub-cells out of the number of absorptions (from the absorption location knowledge) in each sub-cell. From the definition of $N_{\max }$, we see that the maximum amount of memory used to store the absorption locations, is $\propto N_{\max } \times N_{\text {cells }}$. For the typical values used in the numerical tests of Sect 4, i.e. $N_{\max }=10^{2}$ (which corresponds typically to $\sim 10 \%$ of the computing time dedicated to the grid construction) and $N_{\text {cells }}=10^{5}$, this gives a memory of $\approx 80 \mathrm{MB}$ (for floating numbers represented with 8 bytes).

- The algorithm is repeated until the prescribed number of cells $N_{\text {cells }}$ has been reached. At that point, the storage of the absorption locations can be freed.

With this algorithm the propagation of the photon packages, the computation of the radiative equilibrium and the construction of the grid are done at the same time. The reason for this comes from the coupling of these computing phases: the photon package propagation depends on the temperature structure, which depends on the grid temperature which depends on the package propagation, ... As a consequence, the grid is necessarily built while proceeding to the random walks of the photon packages and is not computed before and independently of this random walk. However, this algorithm can be used in two ways:

- the first usage is to proceed in two phases: an initial rough MC simulation (i.e. with a small number of photon package such as $10^{6}$ ) can be done to compute the grid. Then, this grid can be read out and used as a fixed grid in another MC simulation with a more suitable (larger) number of photon packages;

- the second usage arose from the observation that in practice, it appears that proceeding to a unique MC simulation, during which the grid is built during a fraction of the computing time (for the computations presented in this paper $N_{\max }$ was chosen so that this fraction is $\approx 10 \%$ ) provides the same level of accuracy for the results (see Sect. 4). This solution has been adopted for the numerical tests in Sect. 4.

The algorithm for the generation of the temperature grid is controlled by two parameters: $N_{\text {cells }}$ and $N_{\text {max }}$. As could be expected the larger $N_{\text {cells }}$ is, the better is the sampling of the temperature and the better the accuracy (i.e. discrepancy from a reference solution, either theoretical or well established benchmarks) of the results. However, the effect of $N_{\max }$ on the accuracy (if we fix the total number of photon packages used in the simulation) of the final results is not trivial. A detailed numerical study is given in Sect. 4.4.

\subsection{Tree algorithm}

Since we must store information, such as the number of absorptions in each cell, we must be able to determine to which cell a given point $\boldsymbol{r}$ belongs. A significant part of the computation time is dedicated to this task. 
Given $\boldsymbol{r}$, we can proceed in a trivial way, exploring the whole set of cells, and end the search when we find the cell we are in. However, with this simple algorithm, one will have to explore at most $N_{\text {cells }}$, which can end up in a very time consuming search.

To solve this problem, one might want to use a tree algorithm that has been extensively used for instance in the case of $N$-body type codes, widely employed in astrophysics (Barnes \& Hut 1986). In this framework, the cells are ordered in a data tree. The cells contain sub-cells (e.g. in Cartesian coordinates, a cube contains 8 sub-cubes), and are ordered in the tree according to this relation: a given cell points to its sub-cells, themselves pointing to their sub-cells, and so on. By successively determining the cell to which $\boldsymbol{r}$ belongs, climbing down along the tree branches, one explores at most $\log \left(N_{\text {cells }}\right)$, reducing the complexity of the algorithm and the computing time accordingly.

Giving the absorption point $\boldsymbol{r}$ of a photon package, we increment the number of absorptions in the cell we finally indentify, and if greater than $N_{\max }$, as set out in Sect. 3.1, we split this cell into its sub-cells, taking care of where in these new sub-cells the previous absorptions took place.

\section{Numerical tests}

In this section we test the reliability of the grid construction algorithm together with the new temperature determination presented in Sect. (2.4.2) by comparing the results of the MC simulation with the benchmark results computed by Ivezic et al. (1997) in 1D, and from the 2D benchmarks provided by Pascucci et al. (2003). Indeed, there is no exact theoretical result, except for the optically thin grey case neither in $1 \mathrm{D}$ or $2 \mathrm{D}$, for the problem of radiative transfer as described in Sect. 2 against which one could test a numerical method.

The results of Ivezic et al. (1997) were obtained with the help of three codes that agreed together within $0.1 \%$ for the temperatures and the SED. The present numerical tests have been made thoroughly in comparison to the Ivezic et al. (1997) results. Indeed, thanks to the very good agreement of the numerical results in these special 1D cases, the Ivezic et al. (1997) benchmarks are critical numerical tests for a transfer program to compare with.

The Pascucci et al. (2003) 2D benchmarks, however, do not have the excellent numerical agreement between the five codes used for these tests because of the more complex geometry, that of an accretion disc (see below). Still, the differences do not exceed $15 \%$ and $10 \%$ for the temperature and the SED respectively. In spite of these discrepancies, testing our method against these benchmarks will provide information on the ability of the method to face multi-dimensional problems and to reinforce our confidence in our numerical results. In addition, a modification of the Pascucci et al. (2003) accretion disc in the form of a warped disc will be presented in Sect. 5 as an illustration of the 3D capabilities of our method.

\section{1. $1 D$ tests}

These tests are identical to the benchmark problems of Ivezic et al. (1997). We recall here the conditions of the test with the notation of the present paper. The extinction cross-section is given by

$C_{v}^{\mathrm{ext}}= \begin{cases}C_{v_{0}}^{\mathrm{ext}} & \text { for } v \geq v_{0}, \\ \frac{C_{v_{0}}^{\mathrm{ext}}}{2}\left[\left(\frac{v}{v_{0}}\right)+\left(\frac{v}{v_{0}}\right)^{4}\right] & \text { for } v \leq v_{0},\end{cases}$ where $C_{v_{0}}^{\text {ext }}$ is the extinction cross-section at the reference frequency $v_{0}$. For this particular test, $v_{0}=2.99792458 \times 10^{14} \mathrm{~Hz}$ (corresponding to $\lambda=1 \mu \mathrm{m}$ ).

The albedo is defined as

$\gamma_{v}= \begin{cases}\frac{\gamma_{v_{0}}}{2} & \text { for } v \geq v_{0}, \\ \frac{\gamma_{v_{0}}}{1+\left(\frac{v}{v_{0}}\right)^{-3}} & \text { for } v \leq v_{0},\end{cases}$

and the scattering is assumed to be isotropic, i.e. $p_{v}=\frac{1}{4 \pi}$.

With the expressions (9) and (10), two number densities are tested, referred to as test case 1 and 2 in the following,

$n(r)=\left\{\begin{array}{lll}n_{0} & \text { for } R_{\text {in }} \leq r \leq R_{\text {out }} & \text { (test case 1) } \\ n_{0}\left(\frac{R_{\text {in }}}{r}\right)^{2} & \text { for } R_{\text {in }} \leq r \leq R_{\text {out }} & \text { (test case 2) }, \\ 0 & \text { anywhere else } & \text { (both cases) }\end{array}\right.$

where $R_{\text {in }}$ and $R_{\text {out }}$ are respectively the inner and outer radii of the model volume considered.

For each test case, the computation is carried out for three different values, 1, 10 and 100, for the optical depth at frequency $v_{0}, \tau_{v_{0}}$, defined as

$\tau_{v_{0}}=\int_{R_{\text {in }}}^{R_{\text {out }}} n(r) C_{v_{0}}^{\text {ext }} \mathrm{d} r$.

This leads to the three values for $n_{0}$

$n_{0}=(p-1) \frac{\tau_{v_{0}}}{C_{v_{0}}^{\mathrm{ext}} R_{\mathrm{out}}}\left(\frac{R_{\mathrm{out}}}{R_{\mathrm{in}}}\right)^{p}\left\{\left(\frac{R_{\mathrm{out}}}{R_{\mathrm{in}}}\right)^{p-1}-1\right\}^{-1}$,

where $p$ is either 2 or 0 . The value of $R_{\text {in }}$ and $R_{\text {out }}$ are fixed according to Ivezic et al. (1997).

We finally obtain 6 different test cases. The results are shown in Figs. 1 and 2 for test case 1 and 2 respectively. We used $N_{\text {total }}=10^{8}$ photon packages as well as $N_{\text {cell }}=10^{5}$ cells for all the computation presented in this section. $N_{\max }$ has been set to 100 and 200 for test case 1 and 2 respectively. The temperature we consider is sampled in practice on a 3D Cartesian irregular grid. Because of the physical symmetry of the problem, the temperature depends only on $r$, the distance to the centre. The way to obtain the 1D temperatures variation with $r$ out of the $3 \mathrm{D}$ and irregular temperature structure is not canonical. We thus give an exact description of the quantities shown in Fig. 2 in Appendix A.

The largest mean relative differences (as defined in Sect. 4.3) between our results and the benchmarks taken as reference solutions are, in all cases presented in this section, lower than $\sim 1 \%$ and $\sim 2 \%$ for the temperature and the SED respectively.

\section{2. $2 D$ tests}

For these tests we consider the accretion disc density described in Pascucci et al. (2003). The description of the benchmark settings is left to Sect. 5, where we present an application derived from the particular geometry and conditions of the Pascucci et al. (2003) benchmarks.

These benchmarks are 3 optically thick test cases, corresponding to 3 optical depths defined at $0.55 \mu \mathrm{m}, \tau_{0.55 \mu \mathrm{m}}$ in the plane of the disc: 1,10 , and 100. We show in Fig. 3 the comparison of the SED with the output of the code MCTRANSF (Niccolini et al. 2003), for a particular inclination of the disc plane with respect to the line of sight $\left(12.5^{\circ}\right.$ above the disc plane). 

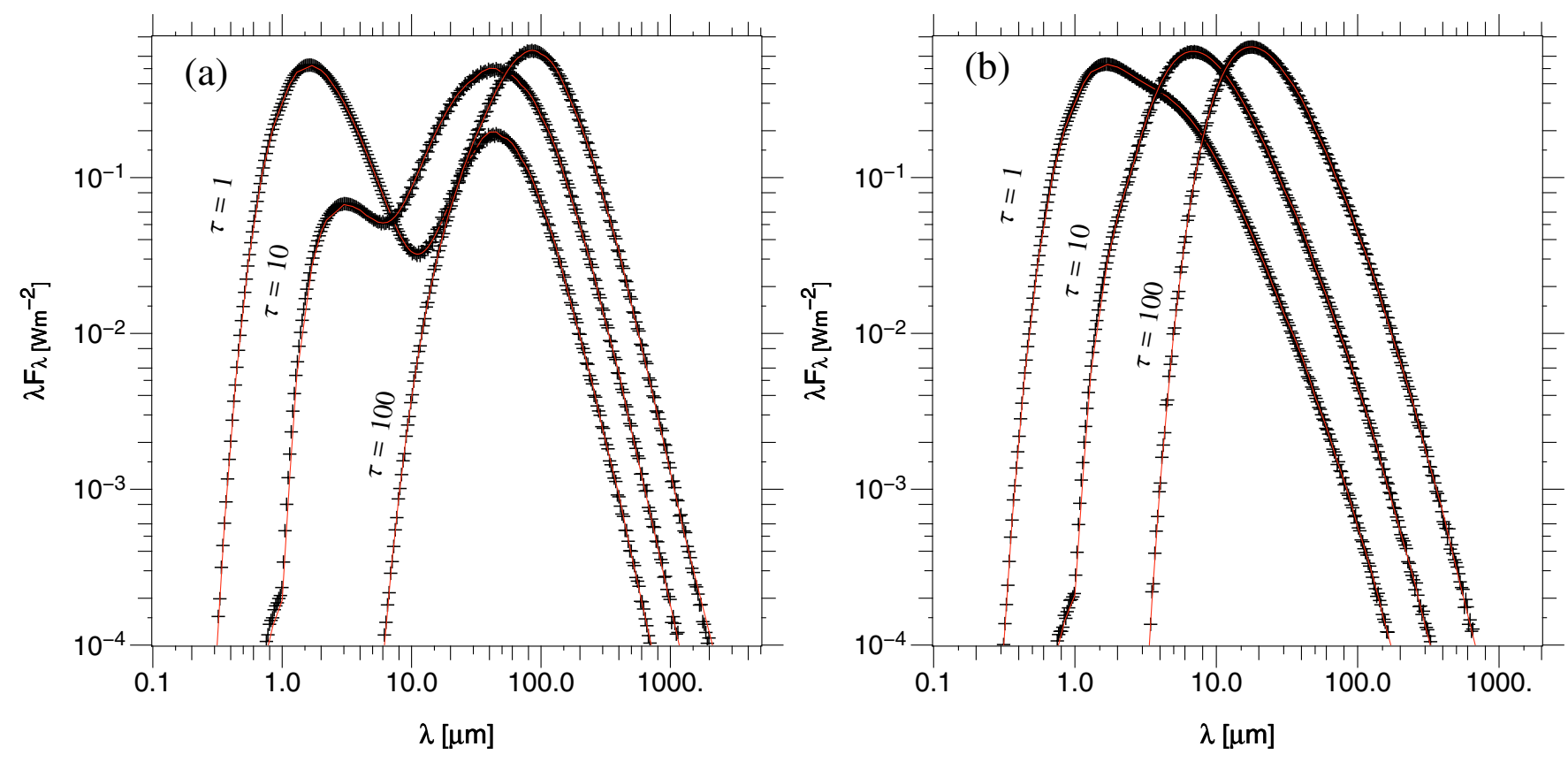

Fig. 1. 1D numerical tests: SED. a) corresponds to test case $0(p=0)$ while b) corresponds to test case $2(p=2)$. The results of the MC simulation are shown by crosses and are obtained with 500 frequency bins (logarithmically spaced). The results from Ivezic et al. (1997) are shown as full lines.
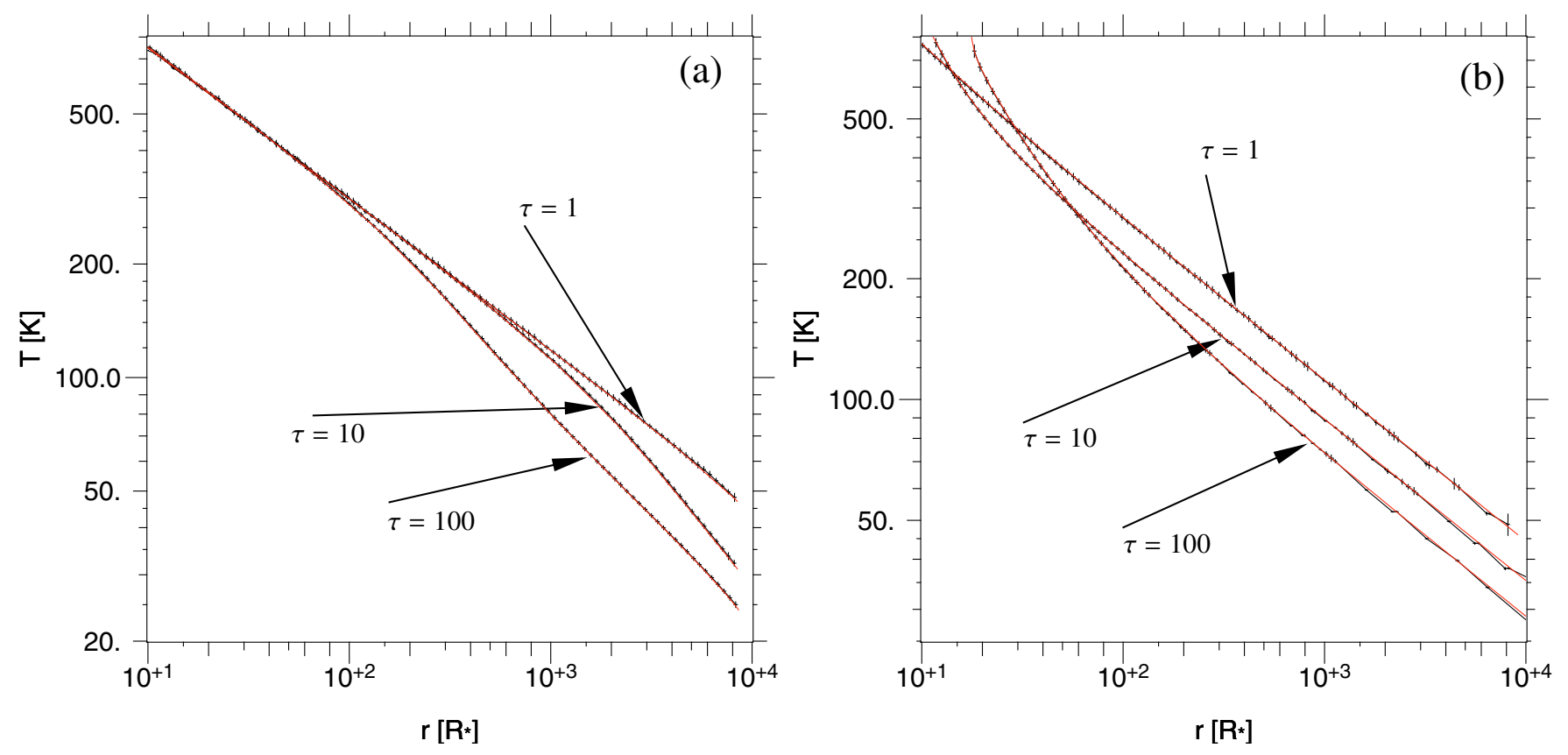

Fig. 2. 1D numerical tests: temperatures. a) corresponds to test case $0(p=0)$ while b) corresponds to test case $2(p=2)$. The $r$ and $T$ values and their corresponding error bars $\sigma_{T_{r}}$ (representing the MC noise, see text) are given by Eqs. (A.2) and (A.4) and are obtained for 100 radial bins (logarithmically spaced). The results from Ivezic et al. (1997) are shown as full lines.

Temperatures (along a radial cut $2.5^{\circ}$ above the disc plane) and SED (for the inclination shown in Fig. 3) obtained in the present work do not differ more than 5\% and 10\% respectively, from MCTRANSF's results. These differences are within the accuracy of the benchmark results (see Pascucci et al. 2003). We used $N_{\text {total }}=5 \times 10^{7}$ photon packages for $\tau_{0.55 \mu \mathrm{m}}=1,10$ and $N_{\text {total }}=5 \times 10^{8}$ for $\tau_{0.55 \mu \mathrm{m}}=100$. We used $N_{\text {cell }}=10^{5}$ and $N_{\max }=100$ in all cases.

\subsection{Temperature and flux estimator qualities}

In this section we present numerical tests of the quality (precision and accuracy) of the temperature and flux estimators as computed from Eqs. (5) and (6).

We defined two quantities: the relative Monte Carlo noise and the relative difference between our results and a reference solution. We take as the reference solution the test case 2 of 


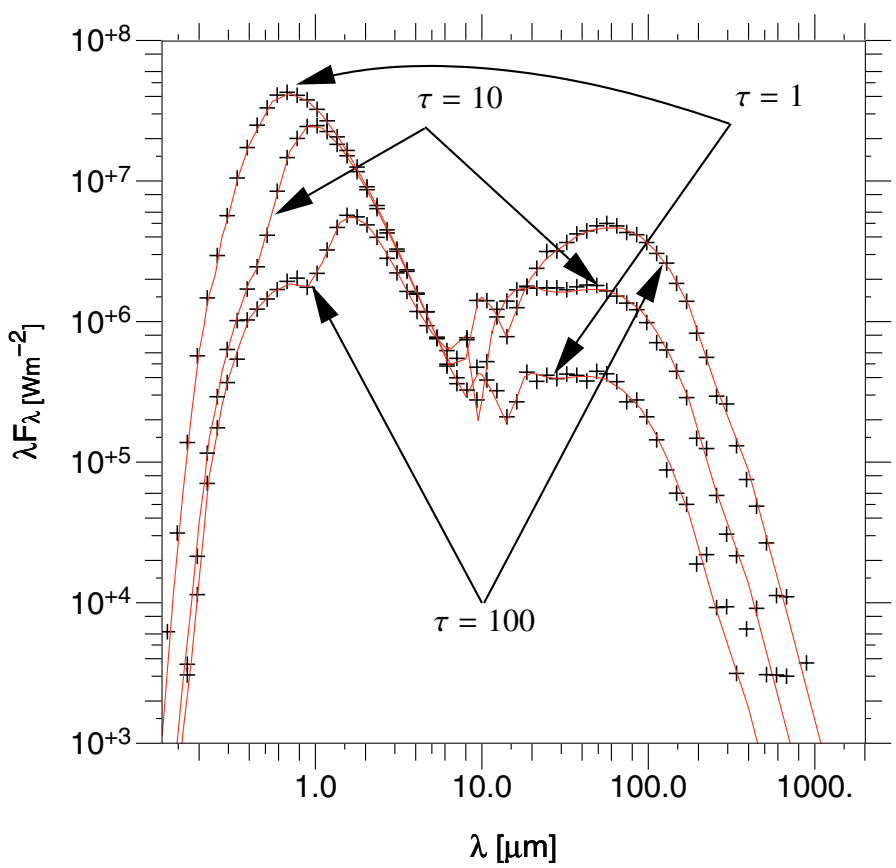

Fig. 3. 2D numerical tests: results for the SED. The results from MCTRANSF, i.e., the 2D benchmarks of Pascucci et al. (2003), are represented as solid lines; the results from out 3D code are represented by crosses. The values of the fluxes are obtained for an arbitrary distance of one stellar radius (see Pascucci et al. 2003).

Sect. 4.1, for $\tau_{v_{0}}=10$. We denote as $\bar{F}_{\lambda}$ and $\bar{T}_{r}$ the reference solutions for the SED and the temperature respectively, where the indexes recall the variation according to the wavelength and the radius. We will refer to our solutions (determined from Eqs. (5) and (6)) as $F_{\lambda}$ and $T_{r}$ for the SED and the temperature respectively. In Appendix A we describe how we estimate the Monte Carlo noise $\sigma_{T_{r}}$ in $T_{r}$ from the 3D temperature structure. We can obtain a Monte Carlo noise $\sigma_{F_{\lambda}}$ estimation for $F_{\lambda}$ by computing the flux for 100 different angular directions and obtain $\sigma_{F_{\lambda}}$ as the root mean square deviation of the values obtained, using the expected spherical symmetry of the results.

Both MC noise and difference to the reference solution vary with the radius and the wavelength. For simplicity, we rather compute single averaged values out of these quantities. We represent in Fig. 4 these averages for the MC noise and the difference to the reference solution as a function of the fraction of photon packages emitted, $\frac{N}{N_{\text {total }}} . N$ is the number of photon packages emitted at some given time during the computation; $N_{\text {total }}$ is the total number of photon packages emitted for the whole computation. We use $N_{\text {total }}=10^{7} ; N$ varies from 1 to $N_{\text {total }}$. Indeed, $\frac{N}{N_{\text {total }}}$ can be seen as the fraction of computing time during a computation for a given $N_{\text {total }}$.

The average values over the radius and the wavelength for $T_{r}$ and $F_{\lambda}$ are denoted \langle\rangle$_{r}$ and \langle\rangle$_{\lambda}$ respectively. The average is weighted by the inverse of the square of the relative Monte Carlo noise, $\left(\frac{T_{r}}{\sigma_{T_{r}}}\right)^{2}$ and $\left(\frac{F_{\lambda}}{\sigma_{F_{\lambda}}}\right)^{2}$ for the temperature and the flux respectively. Indeed with the present method, the Monte Carlo noise of the temperature and the SED vary with $r$ and $\lambda$. For instance for the SED, the MC noise will be larger where the flux values are smaller. The weights are used to counterbalance this effect.

The computation shown in Fig. 4 was done with a fixed temperature grid in order to separate the problem of the temperature and flux determination from the problem of the grid construction. A simulation with $N_{\text {total }}=10^{6}$ photon packages and $N_{\max }=2 \times 10^{2}$ was used to generate this grid.

From Fig. 4 we can see a comparison of the present method and the one from BW. At the end of the computation (i.e. when $\left.N=N_{\text {total }}\right)$ both methods reach roughly the same level of precision (same MC noise) and accuracy (same difference to the reference solution). The MC noise has the same expected behaviour, i.e. $\propto\left(\frac{N}{N_{\text {total }}}\right)^{-1 / 2}$ in both cases and are of comparable magnitudes. However, the differences to the reference solution for the temperature and the SED have different behaviour for the two methods, as could be expected since our method has been designed to reduce significantly these differences. After a few percent of the computation time, our method already gives an accuracy of $\approx 10 \%$ while $\mathrm{BW}$ remains above this for a significantly larger fraction of the computing time $(\approx 60-70 \%)$. This must not be understood as a defect of the BW method. Both methods reach a similar level of precision and accuracy for a given $N_{\text {total }}$, as can be seen from Fig. 6, with comparable computing times (since computation of the expression in Eq. (6) or its BW counterpart are not significantly different). However, with the modifications we propose to compute the temperature, it is possible to have an idea of the final results after a few percent of the total computation time with good accuracy $(\approx 10 \%)$. In practice, while fitting some observational data with a given model, one can have an indication of the validity of the model after a few percent of the total computing time and changed the model accordingly.

\subsection{Effects of $N_{\max }$ on errors (accuracy and precision)}

We show in Fig. 5 the behaviour of $\left\langle\frac{\sigma_{F_{\lambda}}}{F_{\lambda}}\right\rangle_{\lambda}$ (precision) and $\left\langle\frac{\left|F_{\lambda}-\bar{F}_{\lambda}\right|}{\bar{F}_{\lambda}}\right\rangle_{\lambda}$ (accuracy) as a function of $N_{\max }$. The mean values are weighted as described in Sect. 4.3. We choose as a reference solution the test case 2 of Sect. 4.1, for $\tau_{v_{0}}=10$ run with $10^{6}$ photon packages.

For the following discussion we assume a fixed total number of photon packages $N_{\text {total }}$. Increasing $N_{\text {max }}$ reduces the temperature noise (good precision), because it increases the number of absorptions per cell. However, this results in a smaller number of cells during part of the computation time during which the grid is generated, or equivalently a poor sampling of the temperature structure. For instance, if the total number of absorptions in the complete $\mathrm{MC}$ simulation never reach $N_{\max }$, we treat the problem with one cell, resulting in the poorest sampling of the temperature problem.

On the contrary, reducing the $N_{\max }$ value leads to a larger number of cells that could give good accuracy. However, the MC noise increases per cell. It can even alter the accuracy. This larger noise results in poor precision (and thus accuracy) since we can be far from the expected solution.

There is another effect of $N_{\max }$ on the grid construction. The grid is built from the map of absorptions occurring during the simulation. The smaller $N_{\max }$, the faster the grid is generated, but the worse the grid "represents" the map of absorptions. Of course, if we increase $N_{\max }$ (and thus the memory consumption), we obtain a finer representation of the grid, but we spend more time-even the entire computing time-in the worse situation with fewer cells. For a fixed $N_{\text {total }}$, this leads to a poor sampling of the temperature structure.

There is a value for $N_{\max }$ that gives a compromise between large and small $N_{\max }$, i.e. a value for $N_{\max }$ maximising the accuracy of the results as can be seen in Fig. 5. In practice, value of a few hundred proved to be close to the $N_{\max }$ value maximising 

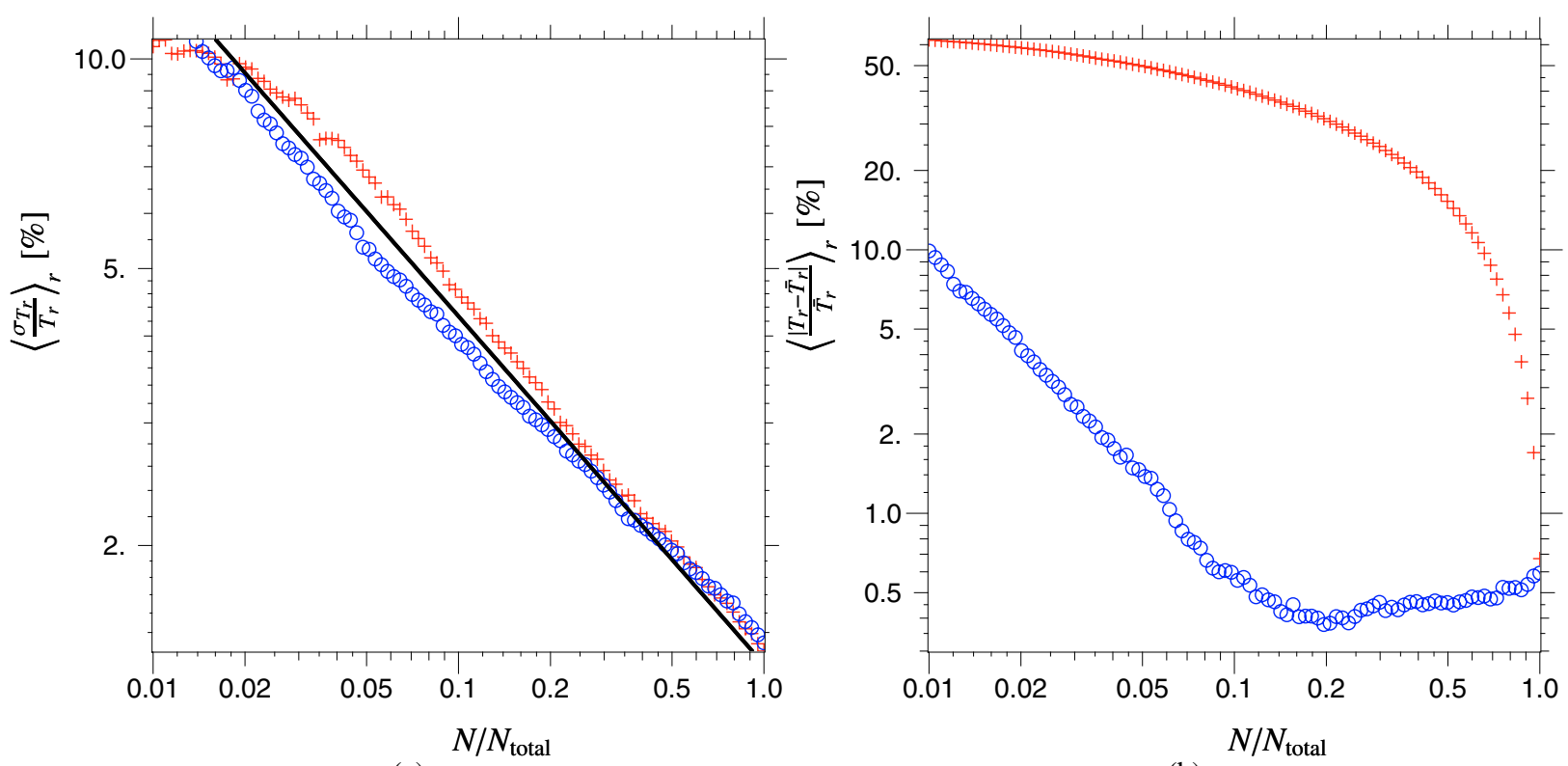

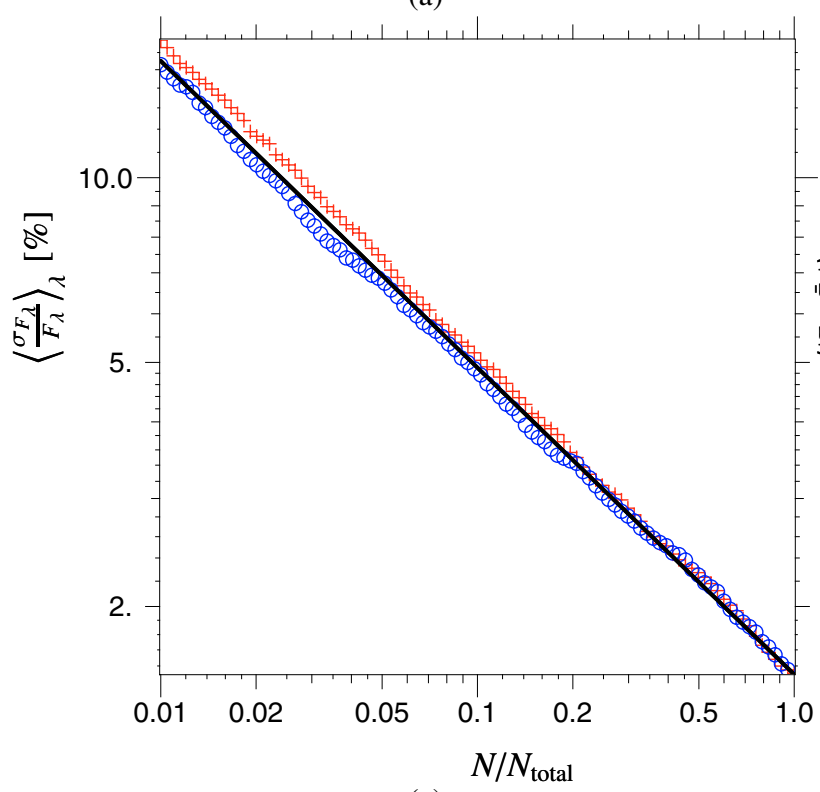

(c)

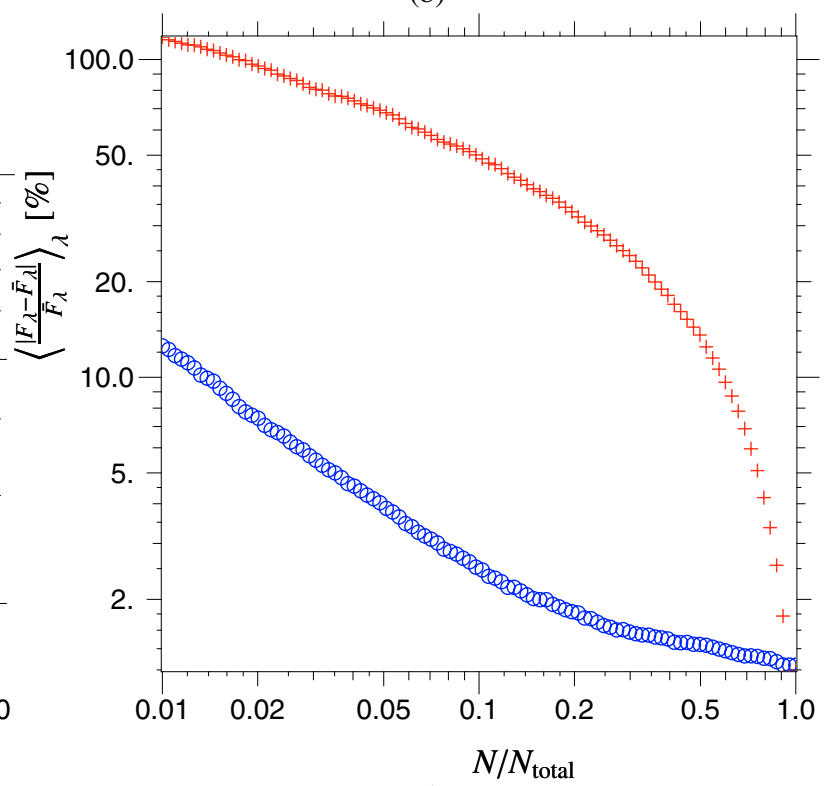

(d)

Fig. 4. Monte Carlo Noise (left) and discrepancy from the benchmark results (right) for the temperature (upper part) and the SED (lower part). All values are relative quantities and expressed in \%. The scaling of each plot is logarithmic on both axis. The circles represent the results obtained from Eqs. (5) and (6), while crosses represent the results obtained using the BW method. The full lines (left plots) show the $\propto\left(\frac{N}{N_{\text {total }}}\right)^{-1 / 2}$ expected dependence of the MC noise.

the accuracy in all cases presented in this work. From Fig. 5 it can also be seen that $N_{\max }$ has a weak influence on the MC noise in the SED. Indeed this noise is determined by the number of photon packages used in the MC simulation.

\subsection{Grid construction algorithm}

With the construction algorithm of the temperature grid (see Sect. 3.1), our purpose is to refine the grid according to the photon package absorption map. The random walks of the photon packages that we use to represent the radiation field are controlled partly by the processes of absorption and re-emission (see Sect. 2.3). These processes depend on the temperature, which is an unknown in our problem. We saw in Sect. 4.3 that with the temperature computation method of Sect. 2.4.2 we approach with a good level of accuracy the final solution for the temperature and the SED in a short fraction (typically a few percent) of the total computing time. We make use of this fast approach to the final solution to build the grid during the sampling of the radiation field, a feature imposed by the algorithm.

There are two ways to use the grid construction. We can either do a first MC simulation from which we obtain solutions to the problem for the temperature structure, the fluxes and particularly for the grid. This grid "represents" the absorptions map to a certain degree of accuracy. The larger $N_{\text {cell }}, N_{\text {max }}$ and $N_{\text {total }}$, the better is this representation. Once this grid is computed, we can proceed to a second simulation making use of our previous knowledge of the grid structure. 


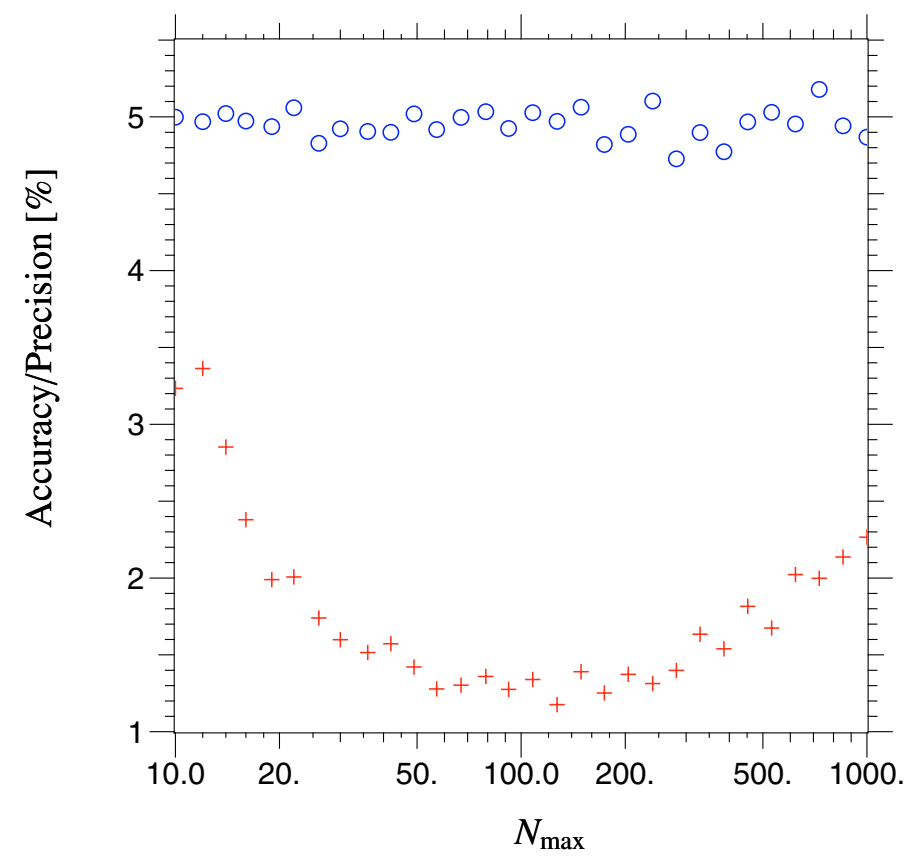

Fig. 5. Choice of $N_{\max }$. The circles represents the quantity $\left\langle\frac{\sigma_{F_{\lambda}}}{F_{\lambda}}\right\rangle_{\lambda}$ (precision) while the crosses represents $\left\langle\frac{\left|F_{\lambda}-\bar{F}_{\lambda}\right|}{\bar{F}_{\lambda}}\right\rangle_{\lambda}$ (accuracy). The abscissa and ordinate scalings are respectively logarithmic and linear.

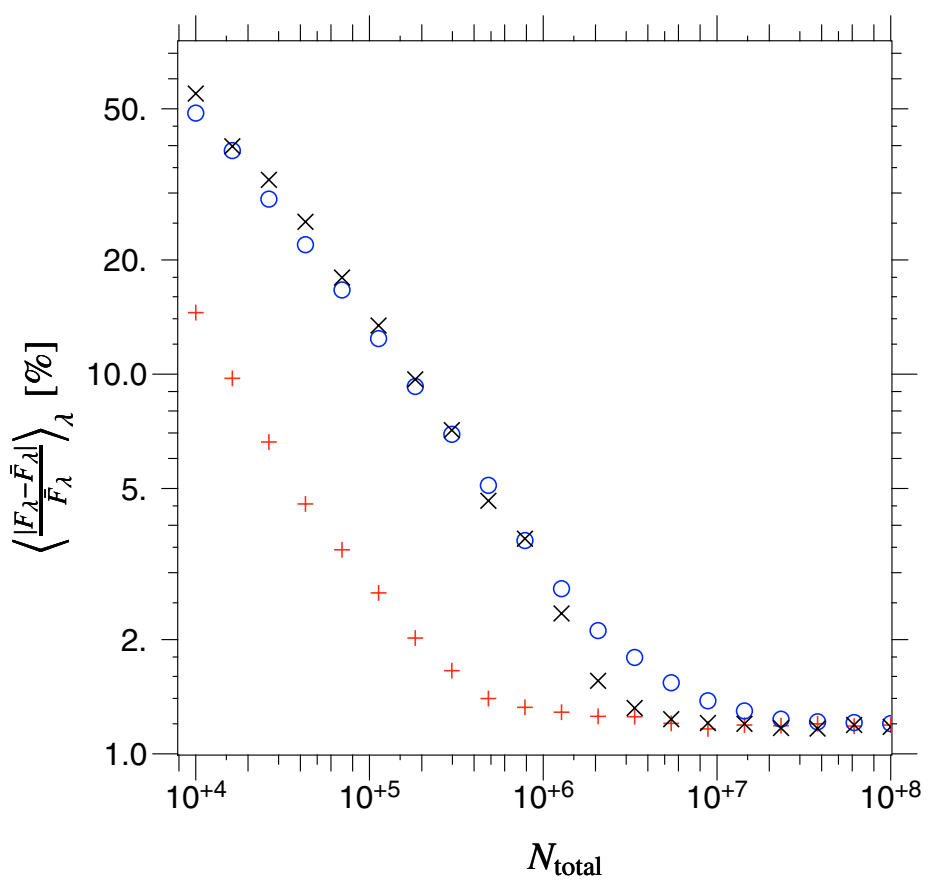

Fig. 6. Accuracy vs. $N_{\text {total }}$. The circles and the plus signs corresponds to the cases where the same grid (pre-computed) is used for all $N_{\text {total }}$ and where the grid is computed during the simulation (see text for more details). The crosses represents the results obtained with the BW method in the case of a pre-computed grid.

The second possibility is to proceed to a unique simulation, dedicating part of the computing time to building the grid, but still making use of the other aspects of the MC simulation: the computation of the temperature and the sampling of the radiation field. In this case, during the time the grid is generated, it is refined during the MC simulation. It is not obvious how to determine to what extent this procedure might affect the accuracy of the final results.
Table 1. Warped disc model parameters.

\begin{tabular}{lcc}
\hline \hline Parameters & Comments & Value \\
\hline$\rho_{0}$ & distance & $500 \mathrm{AU}$ \\
$n_{0}$ & density at $\rho=\rho_{0}$ & $3.15 \times \mathrm{m}^{-3}$ \\
$h_{0}$ & height at $\rho=\rho_{0}$ & $125 \mathrm{AU}$ \\
$p$ & $n$ exponent & -1 \\
$q$ & disc variable height exponent & 1.125 \\
$\alpha$ & $z$-amplitude of the warp & $2 h_{0}$ \\
$\beta$ & must be an integer & 2 \\
$\gamma$ & $\rho$-amplitude of the warp & $\rho_{0}$ \\
\hline
\end{tabular}

To this end, we show in Fig. 6 the quantities $\left\langle\frac{\left|F_{\lambda}-\bar{F}_{\lambda}\right|}{\bar{F}_{\lambda}}\right\rangle_{\lambda}$ (accuracy) as a function of the number of photon packages $N_{\text {total }}$ for the two approaches depicted above. We choose as a reference solution the test case 2 of Sect. (4.1), for $\tau_{\nu_{0}}=10$. The circles correspond to the case for which a grid is previously generated with a previous simulation $\left(N_{\text {total }}=10^{6}, N_{\max }=100\right.$ and $N_{\text {cell }}=10^{5}$ ) and then use for all $N_{\text {total }}$. The crosses show the results when the grid is computed during the MC simulation $\left(N_{\max }=100\right.$ and $\left.N_{\text {cell }}=10^{5}\right)$. We see that the same level of accuracy $(\approx 1 \%)$ is reached in both cases. In practice, the second possibility could be preferred to avoid a prior computation of the grid. It also shows a faster convergence to the final solution.

\section{3D applications: warped discs}

The astrophysical situation where a central source is surrounded by a gaseous/dust disc is very common. Such discs are observed or expected around Young Stellar Objects (YSOs), Planetary Nebulae (PN), Active Galactic Nuclei (AGN), galaxies and X-ray binaries. There are theoretical arguments supporting the presence of warps in these discs. Pringle (1996, 1997) showed that accretion discs are unstable under the effect of a central source radiation pressure and can develop warps. Nayakshin (2005) proposed a warping mechanism for AGN discs due to gravitational effects. Mechanisms involving the magnetic field (e.g. Pfeiffer \& Lai 2004) and irradiation-driven disc wind (e.g. Schandl \& Meyer 1994) have been proposed. There is observational evidence of the presence of a warped disc around $\beta$-Pictoris (see Heap et al. 2000) and the PN NGC 6302 (Matsuura et al. 2005). For PNe, the presence of warps is proposed as a shaping mechanism by Icke (2003) in the case of point symmetric sources. These observations, together with theoretical works, support the idea that warps are common features of astrophysical discs.

The goal of this section is to test the ability of our methods to handle complex geometries. We face the problem of solving the radiative transfer equation through a warped accretion disc with obvious applications in many astrophysical situations.

\subsection{The warped disc model}

We consider the special case of an accretion disc, as described by Chiang \& Goldreich $(1997,1999)$ and taken as benchmarks in the case of passive T-Tauri discs by Pascucci et al. (2003). The dust opacities of these tests are obtained with the Mie theory using Draine \& Lee's (1984) silicate grains of fixed size $0.12 \mu \mathrm{m}$. In this section, we present the results for a warped version of the disc used in the Pascucci et al. (2003) benchmarks and compare them to the non-warped disc results. The idea of starting from the basis of the Pascucci et al. (2003) work is to provide a simple 


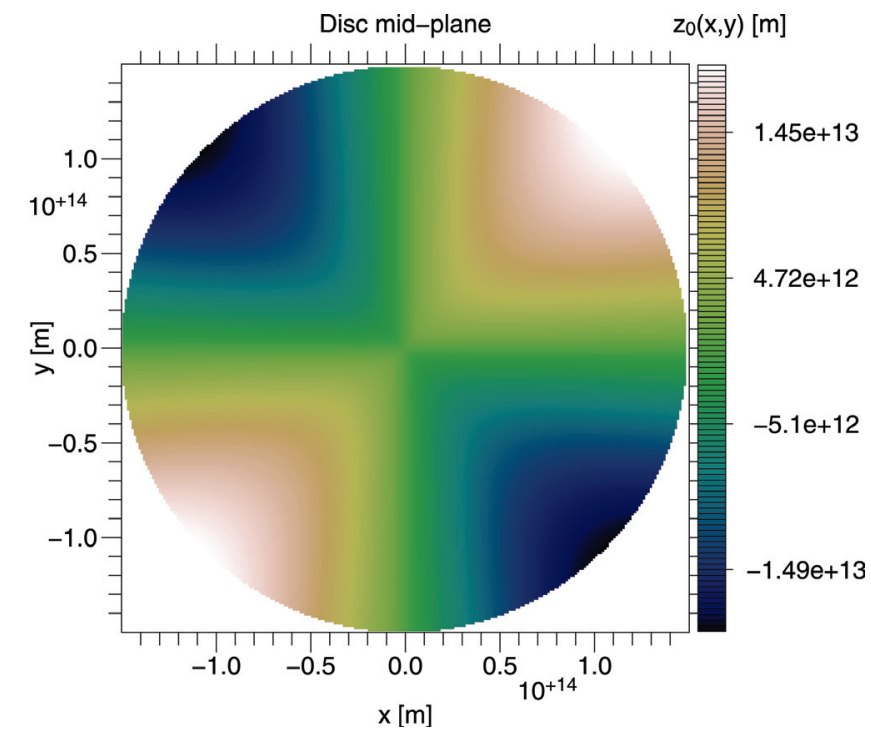

Fig. 7. Warped disc mid-plane. $z_{0}$ is represented as a function of $x$ and $y$, the Cartesian coordinates.

situation against which radiative transfer programs can be easily tested.

The disc number density, $n$, is given by

$n(\rho, \varphi)=n_{0}\left(\frac{\rho}{\rho_{0}}\right)^{p} \exp -\frac{\pi}{4}\left[\frac{z-z_{0}(\rho, \varphi)}{h_{0}\left(\frac{\rho}{\rho_{0}}\right)^{q}}\right]^{2}$,

where $h_{0}$ and $n_{0}$ are respectively the height and the number density of the disc at a distance $\rho_{0}$ from the centre of the star. $\rho, \varphi$ and $z$ are the cylindrical coordinates.

The variation of the disc mid-plane surface, depicting the warp, is described by the function $z_{0}$, taken to be

$z_{0}(\rho, \varphi)=\alpha \sin (\beta \varphi) \frac{2}{\pi} \arctan \left(\frac{\rho}{\gamma}\right)$.

These kind of warp is purely descriptive but provide a simple analytical law that one can easily implement for testing purpose. Note that $z_{0}$ presents a point-like symmetry. Putting $z_{0}=0$ for all $z$ and $\varphi$ in Eq. (14) we obtain the Pascucci et al. (2003) disc geometry. In Table 1 we gathered the model parameters; except for $\alpha, \beta$ and $\gamma$ which are new, the parameters are those of Pascucci et al. (2003). Note that $n_{0}=3.15 \mathrm{~m}^{-3}$ corresponds to $\tau_{0.55 \mu \mathrm{m}}=100$.

In Fig 7 we have represented $z_{0}$ as a function of the Cartesian coordinates $x$ and $y$ for the parameters $\alpha, \beta$ and $\gamma$ given in Table 1.

\subsection{Predicted observational results}

In Fig. 8 we show the computed photometric results of the warped and non-warped disc models. Because of the geometry of the warped disc, the optical depth may have strong variations with the direction of the line of sight. These variations are reflected in the SED of the object. For the warp considered in our example, one obtains a strong deviation from the non-warped disc model (see Fig. 8) for instance along the line of sight specified by $\theta=60^{\circ}$ and $\varphi=45^{\circ}$ (the spherical coordinates). As we can see the warps may lead to large photometric differences. However, despite these large discrepancies, given an observed SED it is not clear how to determine which kind of disc geometry

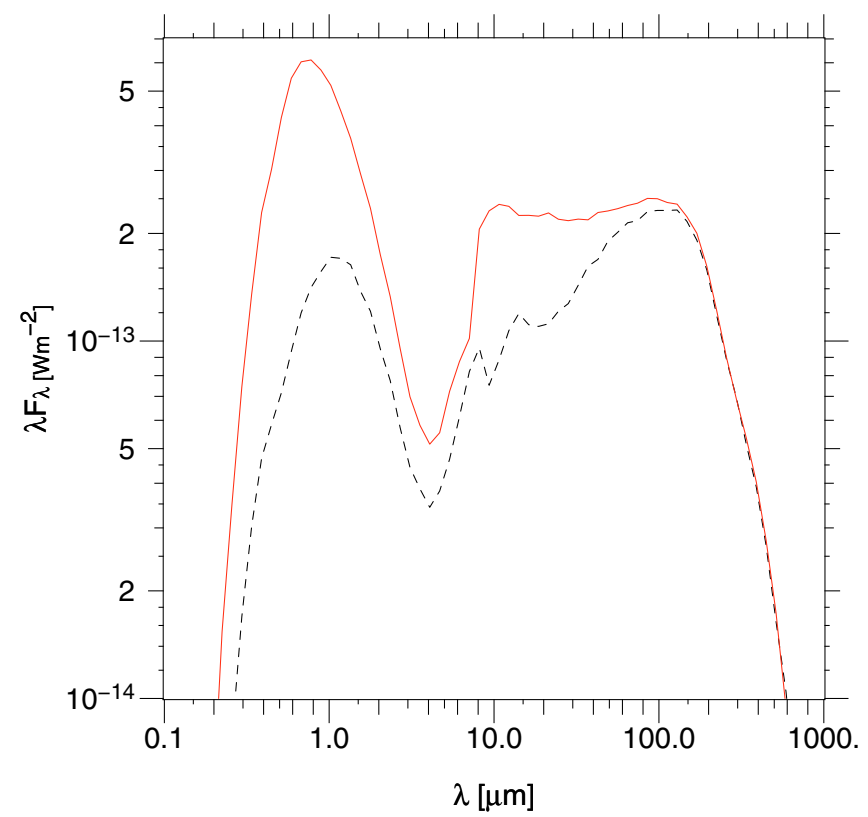

Fig. 8. SED comparison of warped and non-warped discs. The fluxes have been scaled to a distance of $150 \mathrm{pc}$ corresponding to Taurus objects. The results are represented for a given line of sight defined by the spherical coordinates $\theta=60^{\circ}$ and $\varphi=45^{\circ}$. The dash lines represent the warped disc results, while the solid lines represent the non-warped counterpart.

is present. Indeed, the SED by itself does not provide enough information, and its shape depends on many other critical factors: e.g. optical depth, dust grain optical properties, etc. Furthermore, we found no significant difference for discs viewed almost poleone. The difference appears only when the line of sight enters the disc. Thus, although large discrepancies between both geometries are expected, it is almost impossible to determined whether a disc is warped with the only constraints provided by the SED.

For the image production we use a standard procedure for Monte Carlo methods described for instance by Fischer et al. (1994). The reason is that the propagation algorithm we use to compute the temperatures and build the grid is not efficient in generating images and the algorithm initially proposed by Cashwell \& Everett (1959) and described for instance in Gordon et al. (2001) known as "forced first scattering" is preferred.

The differences between both models become obvious from the images. In Fig. 9 we present the results obtained for a given inclination of the line of sight (see caption of Fig. 9) with respect to the disc mid-planes. We choose to compute the images at $10 \mu \mathrm{m}$, a wavelength contained in the bandwidth of the Mid-InfrareD Instrument (MIDI, Leinert \& Graser 1998) of the Very Large Telescope Interferometer (VLTI). Through this wavelength we probe the hot dust emission, i.e. the inner regions of the circumstellar disc. Note that the images presented in this section are barely resolved by MIDI whose angular resolution goes down to $10^{-2}$ arcsec.

At $10 \mu \mathrm{m}$, we see the inner cavity of the accretion disc through the hot $\left(\$ 10^{3} \mathrm{~K}\right)$ dust emission heated by the central star. In Fig. (9a), we show the computed images for the non-warped disc. The shape of this image is elliptical, as could be expected since the inner cavity is spherical. Of course, the shape of the ellipse depends on the inclination of the line of sight with respect to the disc plane. In this case the observer is $35^{\circ}$ above the disc plane, with the bottom part (corresponding to the lower part in 


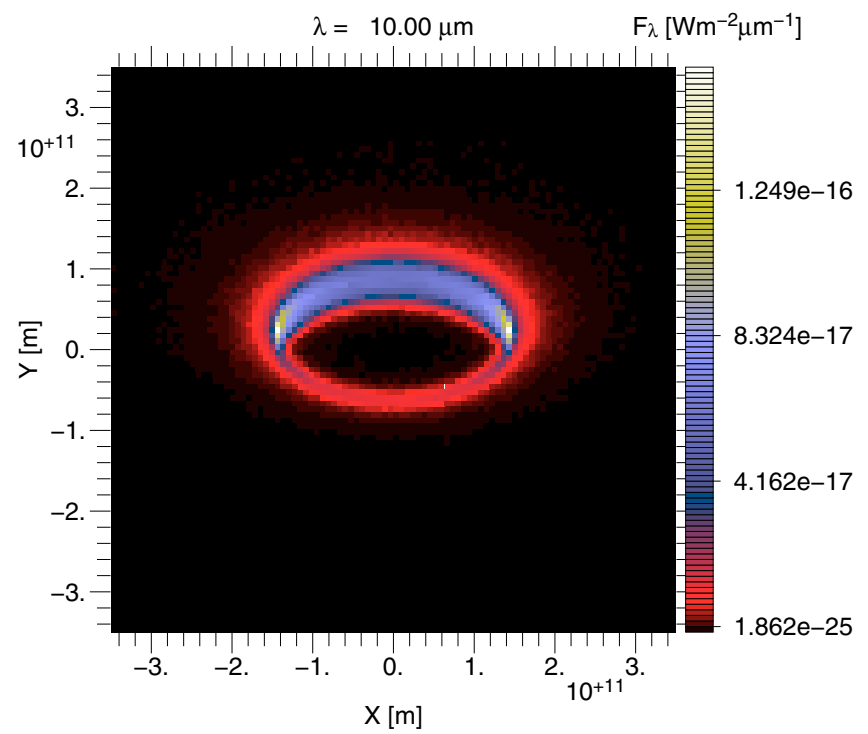

(a)

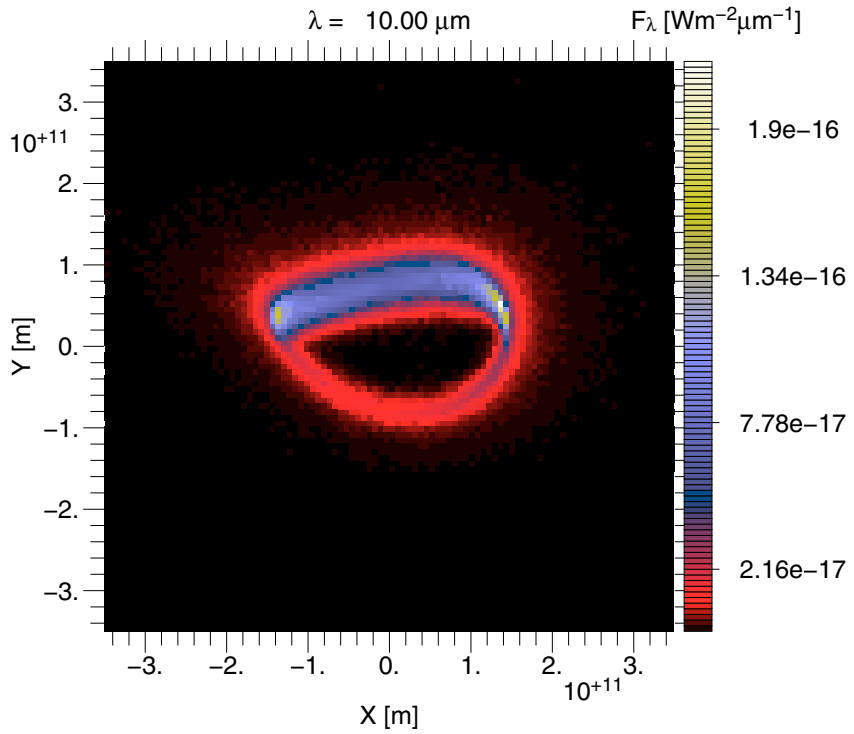

(b)

Fig. 9. Image computed at $10 \mu \mathrm{m}$ for the warped a) and non-warped b) disc. The fluxes have been scaled to a distance of $150 \mathrm{pc}$ corresponding to Taurus objects and the values are given per pixel. The image angular size is 31 mas with a pixel size of 0.31 mas $\times 0.31$ mas. These images correspond to a line of sight in a direction given by the spherical coordinates $\theta=55^{\circ}$ and $\varphi=110^{\circ}$. The $z$ axis would be the axis of symmetry of the non-warped version of the disc. The warped and non-warped disc images have been obtained with $4 \times 10^{8}$ photon packages. The non-warped disc image has been averaged according to the axi-symmetry of the problem.

the image) of the disc oriented towards the observer. On the top of the image is an enhanced emission that traces the disc surface (since those regions of the disc are optically thick) heated up by the star, emitted from the top part of the disc (far away from the observer). This emission cannot be seen on the bottom part of the image. Of course, the emissivity of the disc surface is the same in both parts because of the disc symmetry. However, since the projected (perpendicular to the line of sight) emitting surface is smaller for the bottom than for the top part, the latter appears brighter.

From the warped version of the disc in Fig. 9b, we see that the geometry of the disc (see Fig. 7) is reflected in the image. While the non-warped version looks like an ellipse for any orientation of the disc on the plane of the sky, this is no longer the case for the warped disc: one can clearly distinguish both geometries for any orientation of the disc (expect if viewed pole-on). As for the non-warped disc, the intensity of the emission is proportional to the projection of the emitting surface, since we deal with the optically thick regions of the disc. This projected emitting surface varies with the height of the disc mid-plane represented in Fig. 7 and is related directly to the disc warp. However, not only the surface exposed to the observer varies in that case. The projected surface exposed to the central heating source varies as well, leading to a variation in the inner disc temperatures. For the non-warped disc, one could recover the disc geometry (scale height, flaring ... ) from the projected surface since the emission is axi-symmetric. If the disc is warped, the temperature is not longer axi-symmetric, and the intensities reflect the temperature variations as well and so the radiative transfer must be solved to characterise the warp geometry.

\section{Conclusions}

One of the natural by-products of a Monte Carlo simulation used to solve the problem of radiative transfer is the amount of absorption (per unit volume) of the photon packages in the dusty medium. Using this information, we proposed a method to construct an adaptive grid that allows one to sample full 3D problems with complex and arbitrary geometries. The basis of the algorithm is simple: the grid is refined when a certain number of absorptions per cell is reached. We showed that it is possible to generate the grid during the photon package propagation and still obtain the same level of accuracy and precision.

For the temperature determination we used a modified version of the BW method based on a faster estimator of the temperature of the dusty medium. With such an estimator, we can obtain in a short fraction of the total computing time (typically a few percent) some fairly accurate results (flux and temperature) before the end of the computation. This method has been validated by numerical tests. We analysed the situation where more than one photon package source is considered in a Monte Carlo method, and shown that in that case, special handling of the selfabsorption within the cells must be performed, otherwise errors occur in the temperature determination.

A computer program making use of the methods (for the temperature determination and the grid construction) presented in this paper has been tested against 1D (Ivezic et al. 1997) and 2D (Pascucci et al. 2003) benchmark problems. The program results are in good agreement with these reference solutions.

To test the ability of the radiative transfer program based on the present work to treat complex geometries, we investigated the observational consequence of warps in accretion discs. We found that only imaging can distinguish between a warped and a non-warped geometry. The images of the warped disc depends on this special geometry and also on the temperature of the disc. The resolution of the radiative transfer equation is necessary to determine the complex temperature structure implied by the warps.

We used a simple Monte Carlo scheme to sample the radiation field. We plan to use more complex Monte Carlo methods making use of properly weighted photon packages (see Juvela 2005 , for instance) together with the techniques presented in this 
paper to determine the temperature structure and build the temperature grid.

Acknowledgements. We thank our referee Mika Juvela for his constructive remarks. Many thanks to Jean François Desmurs, Anne Dutrey, Javier GraciáCarpio, Stéphane Guilloteau, Patrick de Laverny, Bruno Lopez, and Olga Suarez for fruitful discussions and/or financial support. We thank Zeljko Ivezic for providing us with his benchmarks results.

This work has been partly supported by a Lavoisier fellowship from the French Foreign Affairs ministry and by the Observatorio Astronómico Nacional (Spain). This work is dedicated to Julie.

\section{Appendix A: 1D temperatures}

We present how we compute 1D temperature profiles out of the 3D Cartesian grid.

The radial range, from $R_{\text {in }}$ to $R_{\text {out }}$, is divided in $N_{r}$ logarithmically spaced bins between the $N_{r}+1$ points $r_{i}$, defined as

$r_{i}=\exp \left[\frac{\log \left(R_{\mathrm{out}} / R_{\mathrm{in}}\right)}{N_{r}} i+\log R_{\mathrm{in}}\right] \quad$ for $i=0, \ldots, N_{r}$.

The $i$ th radial bins is defined from Eq. (A.1) as the interval $\left[r_{i}, r_{i+1}\right)$.

If we note $r_{i}^{k} T_{i}^{k}$ the $N_{i}$ distances (from the centre) and the temperatures of the cells that belongs to bin $i$, we can compute a mean radial distance $\langle r\rangle_{i}$ and a mean temperature $\langle T\rangle_{i}$ for this particular bin with

$$
\begin{aligned}
\langle r\rangle_{i} & =\frac{1}{N_{i}} \sum_{k=0}^{N_{i}} r_{i}^{k}, \\
\langle T\rangle_{i} & =\frac{1}{N_{i}} \sum_{k=0}^{N_{i}} T_{i}^{k} .
\end{aligned}
$$

We can also compute the standard deviations of these values with

$$
\begin{aligned}
{\left[\sigma_{i}(r)\right]^{2} } & =\frac{1}{N_{i}-1} \sum_{k=0}^{N_{i}}\left(r_{i}^{k}-\langle r\rangle_{i}\right)^{2} \\
{\left[\sigma_{T_{r}}\right]^{2} } & =\frac{1}{N_{i}-1} \sum_{k=0}^{N_{i}}\left(T_{i}^{k}-\langle T\rangle_{i}\right)^{2} .
\end{aligned}
$$

These standard deviations are our estimators of the deviations of the $r$ and $T$ values due to the stochastic nature of the MC simulation.

\section{References}

Baes, M., Stamatellos, D., Davies, J. I., et al. 2005, New Astron., 10, 523 Barnes, J., \& Hut, P. 1986, Nature, 324, 446

Bjorkman, J. E., \& Wood, K. 2001, ApJ, 554, 615

Cashwell, E. D., \& Everett, C. J. 1959, A Practical Manual on the Monte Carlo Method for Random Walk Problems (New York: Pergamon Press)

Chiang, E. I., \& Goldreich, P. 1997, ApJ, 490, 368

Chiang, E. I., \& Goldreich, P. 1999, ApJ, 519, 279

de Laverny, P., \& Mékarnia, D. 2004, A\&A, 428, L13

Draine, B. T., \& Lee, H. M. 1984, ApJ, 285, 89

Fischer, O., Henning, T., \& Yorke, H. W. 1994, A\&A, 284, 187

Gordon, K. D., Misselt, K. A., Witt, A. N., \& Clayton, G. C. 2001, ApJ, 551, 269

Harries, T. J., Monnier, J. D., Symington, N. H., \& Kurosawa, R. 2004, MmRAS, 350,565

Heap, S. R., Lindler, D. J., Lanz, T. M., et al. 2000, ApJ, 539, 435

Icke, V. 2003, A\&A, 405, L11

Ivezic, Z., Groenewegen, M. A. T., Men'shchikov, A., \& Szczerba, R. 1997, MNRAS, 291, 121

Juvela, M. 2005, A\&A, 440, 531

Juvela, M., \& Padoan, P. 2003, A\&A, 397, 201

Lefevre, J., Bergeat, J., \& Daniel, J.-Y. 1982, A\&A, 114, 341

Lefevre, J., Daniel, J.-Y., \& Bergeat, J. 1983, A\&A, 121, 51

Leinert, C. \& Graser, U. 1998, in Astronomical Interferometry, ed. R. D. Reasenberg, Proc. SPIE, 3350, 389

Lopez, B., Mekarnia, D., \& Lefevre, J. 1995, A\&A, 296, 752

Lucy, L. B. 1999, A\&A, 344, 282

Matsuura, M., Zijlstra, A. A., Molster, F. J., et al. 2005, MNRAS, 359, 383

Mattila, K. 1970, A\&A, 9, 53

Misselt, K. A., Gordon, K. D., Clayton, G. C., \& Wolff, M. J. 2001, ApJ, 551, 277

Nayakshin, S. 2005, MNRAS, 359, 545

Niccolini, G., Woitke, P., \& Lopez, B. 2003, A\&A, 399, 703

Pascucci, I., Wolf, S., Steinacker, J., et al. 2003, A\&A

Pfeiffer, H. P., \& Lai, D. 2004, ApJ, 604, 766

Pringle, J. 1997, MNRAS, 292, 136

Pringle, J. E. 1996, MNRAS, 281, 357

Roark, T., Roark, B., \& Collins, G. W. 1974, ApJ, 190, 67

Schandl, S., \& Meyer, F. 1994, A\&A, 289, 149

Watson, A. M., \& Henney, W. J. 2001, Rev. Mex. Astron. Astrofis., 37, 221

Witt, A. N. 1977, ApJS, 35, 1

Wolf, S., \& D’Angelo, G. 2005, ApJ, 619, 1114

Wolf, S., Henning, T., \& Stecklum, B. 1999, A\&A, 349, 839 